\title{
Update on drug-repurposing: is it useful for tackling antimicrobial resistance?
}

\author{
Grace Kaul ${ }^{1}$, Manjulika Shuklaํ․ Arunava Dasgupta ${ }^{1}$ \& Sidharth Chopra*,1 iD \\ ${ }^{1}$ Division of Microbiology, CSIR-Central Drug Research Institute, Sector 10, Janakipuram Extension, Sitapur Road, \\ Lucknow-226031, Uttar Pradesh, India \\ *Author for correspondence: Tel.: + 91522277 2450; ext. 4431; Fax: +91 522277 1941; skchopra007@gmail.com
}

\begin{abstract}
'There are several advantages to drug repurposing including leveraging pre-existing safety and clinical data to skip extensive Phase I safety studies and enter clinical trials at Phase Ila, which not only positively impacts development times and costs but also negates risks"
\end{abstract}

First draft submitted: 2 May 2019; Accepted for publication: 17 May 2019; Published online: 25 July 2019

Keywords: antimicrobial resistance $\bullet$ drug discovery $\bullet$ drug repurposing

Antimicrobial resistance (AMR) has been globally recognized as a threat to human health and healthcare systems [1]. In fact, AMR is estimated to be responsible for over 700,000 deaths annually while costing the global economy US $\$ 100$ trillion by 2050 [2]. Concomitant with the unrelenting increase in AMR, conventional drug discovery and development has failed to populate the antimicrobial pipeline with new drugs targeting drug-resistant pathogens, leading to a severe crunch in effective therapeutic options available to attending physicians. In this context, drug repurposing, defined as gaining approval for treatment of another indication for an already approved drug, offers an exciting complementary alternative to rapidly identify already approved drugs exhibiting antimicrobial activity [3]. This approach is immensely necessary since most of the major pharmaceutical companies have exited antimicrobial discovery citing unfavorable economic returns despite the persistent rise of AMR, thus leaving the onus of the antimicrobial discovery to academia/start-up companies [4]. This lack of leadership, coupled with the sluggish pace of conventional drug discovery ( $\sim 20$ years for a drug to hit the market) and exorbitant costs ( $\sim$ US $\$ 5$ billion), necessitate increased utilization of alternative, cheaper strategies such as drug repurposing to expeditiously populate the antibiotic discovery pipeline and allow us to tackle rapidly overwhelming threats such as AMR.

There are several advantages to drug repurposing including leveraging pre-existing safety and clinical data to skip extensive Phase I safety studies and enter clinical trials at Phase IIa, which not only positively impacts development times and costs but also negates risks [5]. In addition, the ability to utilize already existing synthesis procedures and potentially increase the patent life of the drug in question, increases the chances of a successful new drug application, thus enabling the drug to enter the market faster [6]. In fact, classical examples of drug repurposing are sildenafil (originally intended to treat hypertension but repurposed for treatment of erectile dysfunction), thalidomide (originally intended for morning sickness but repurposed for treatment of leprosy) and bupropion (originally developed for depression but repurposed for smoking cessation) among others [6].

In the context of repurposing drugs for antimicrobial activity, there has been increasing reports of approved drugs being identified for their antimicrobial activity. Some of the more prominent examples include auranofin, an antirheumatoid arthritis drug, which was granted an orphan drug status in 2012 by US FDA, repurposed as a broad spectrum antimicrobial and has undergone a clinical trial for the treatment of gastrointestinal protozoa (NCT02736968) [7]; chlorocyclizine, an anti-allergic as an antiviral [8]; eflornithine, an antitumor agent repurposed for the treatment of human African trypanosomiasis [9]; miltefosine, another antitumor agent used to target visceral leishmaniasis [10]; niclosamide, an anthelminthic drug repurposed for antiviral and antibacterial activity [11,12]; pentamidine, an antiprotozoal repurposed as an antibacterial [13]; sertraline, an antidepressant as an antifungal [14]; tamoxifen, an antitumor as an antifungal [15]; ivacaftor, an anticystic fibrosis drug as an antibacterial [16]; DPIC, an nitric oxide synthase inhibitor as a broad spectrum antibacterial [17,18]; disulfiram, an anti-alcoholic drug repurposed as an antibacterial [19,20]; and ebelsen, repurposed as an antibacterial [21], among others. 
Despite these positive efforts, there are significant hurdles encountered in a successful translational implementation of repurposing drugs for antimicrobial activity such clinical pharmacology, pharmacokinetics, high cost of clinical trials and intellectual property rights [22]. This is since antimicrobials are typically dosed at a far higher level than nonantibiotic drugs, thus potentially impacting toxicity and adverse events. Additionally, due to the higher concentrations tested for antimicrobial activity, the original pharmacokinetic profiles might also fall short for the new application particularly for half-life requirements, tissue distribution and plasma protein binding being the typical culprits. Finally, the high financial cost of the clinical trials remains a major roadblock as most repurposed drugs are in the 'generic' stage or at the end of their patent lifetime, thus most pharmaceutical companies are least interested in funding clinical trials. The lack of funding and interest from big pharmaceutical companies has the potential to limit successful translational aspects. One of the potential ways out is to fund the trials through public funding sources or undergo smaller clinical trials [23]. Due to these limitations, often the repurposed compound provides a new chemical lead scaffold for the drug discovery process and must undergo the conventional drug discovery paradigm, which is expensive, time-consuming and unresponsive to rapidly emerging threats like AMR.

Taken together, drug repurposing is a very necessary and pragmatic approach to increasingly identify antimicrobial activities in several approved drugs. This requires the cooperation and goodwill of big pharmaceutical companies for its successful transition to the clinic and can positively impact the identification of new antimicrobials targeting drug-resistant pathogens, a highly unmet need of the healthcare community globally.

\section{Financial \& competing interests disclosure}

G Kaul acknowledges DST-INSPIRE, India for her fellowship while M Shukla thanks University Grants Commission, India. This manuscript bears 9847 as CSIR-CDRI communication number. The authors have no other relevant affiliations or financial involvement with any organization or entity with a financial interest in or financial conflict with the subject matter or materials discussed in the manuscript apart from those disclosed.

No writing assistance was utilized in the production of this manuscript.

\section{References}

1. World Health Organization. Antimicrobial resistance. www.who.int/antimicrobial-resistance/en/

2. Review on Antimicrobial Resistance. https://amr-review.org/

3. Farha MA, Brown ED. Drug repurposing for antimicrobial discovery. Nat. Microbiol. 4, 565-577 (2019).

4. The Pharmaceutical Journal. Why are there so few antibiotics in the research and development pipeline? www.pharmaceutical-journal.com/news-and-analysis/features/why-are-there-so-few-antibiotics-in-the-research-and-develop ment-pipeline/11130209.article?firstPass=false

5. Kepplinger EE. FDA's expedited approval mechanisms for new drug products. Biotechnol. Law Rep. 34(1), 15-37 (2015).

6. Ashburn TT, Thor KB. Drug repositioning: identifying and developing new uses for existing drugs. Nat. Rev. Drug Discov. 3(8), 673-683 (2004).

7. Debnath A, Parsonage D, Andrade RM et al. A high-throughput drug screen for Entamoeba histolytica identifies a new lead and target. Nat. Med. 18, 956-960 (2012).

8. He S, Lin B, Chu V et al. Repurposing of the antihistamine chlorcyclizine and related compounds for treatment of hepatitis C virus infection. Sci. Transl. Med. 7(282), 282 ra49 (2015).

9. Simarro PP, Franco J, Diarra A, Postigo JA, Jannin J. Update on field use of the available drugs for the chemotherapy of human African trypanosomiasis. Parasitology 139, 842-846 (2012).

10. Smorenburg CH, Seynaeve C, Bontenbal M et al. Phase II study of miltefosine 6\% solution as topical treatment of skin metastases in breast cancer patients. Anticancer Drugs 11(10), 825-828 (2000).

11. Xu M, Lee EM, Wen $\mathrm{Z}$ et al. Identification of small-molecule inhibitors of Zika virus infection and induced neural cell death via a drug repurposing screen. Nat. Med. 22, 1101-1107 (2016).

12. Imperi F, Massai F, Ramachandran Pillai C et al. New life for an old drug: the anthelmintic drug niclosamide inhibits Pseudomonas aeruginosa quorum sensing. Antimicrob. Agents Chemother. 57, 996-1005 (2013).

13. Stokes JM, MacNair CR, Ilyas B et al. Pentamidine sensitizes Gram-negative pathogens to antibiotics and overcomes acquired colistin resistance. Nat. Microbiol. 6(2), 17028 (2017).

14. Villanueva-Lozano H, Treviño-Rangel RJ, González GM et al. Clinical evaluation of the antifungal effect of sertraline in the treatment of cryptococcal meningitis in HIV patients: a single Mexican center experience. Infection 46(1), 25-30 (2018).

15. Krysan DJ, Didone L. A high-throughput screening assay for small molecules that disrupt yeast cell integrity. J. Biomol. Screen. 13, 657-664 (2008). 
16. Thakare R, Singh AK, Das S et al. Repurposing Ivacaftor for treatment of Staphylococcus aureus infections. Int. J. Antimicrob. Agents 50(3), 389-392 (2017).

17. Pandey M, Singh AK, Thakare R et al. Diphenyleneiodonium chloride (DPIC) displays broad-spectrum bactericidal activity. Sci. Rep. 7(1), 11521 (2017).

18. Singh AK, Thakare R, Karaulia P et al. Biological evaluation of diphenyleneiodonium chloride (DPIC) as a potential drug candidate for treatment of non-tuberculous mycobacterial infections. J. Antimicrob. Chemother. 72(11), 3117-3121 (2017).

19. Das S, Garg T, Chopra S, Dasgupta A. Repurposing disulfiram to target infections caused by non-tuberculous mycobacteria. J. Antimicrob. Chemother. doi:10.1093/jac/dkz018 (2019) (Epub ahead of print).

20. Thakare R, Shukla M, Kaul G et al. Repurposing disulfiram for treatment of Staphylococcus aureus infections. Int. J. Antimicrob. Agents (53(6), 709-715 (2019).

21. Thangamani S, Younis W, Seleem MN. Repurposing ebselen for treatment of multidrug-resistant staphylococcal infections. Sci. Rep. 5 , 11596 (2015).

22. Oprea TI, Overington JP. Computational and practical aspects of drug repositioning. Assay Drug Dev. Techn. 13, 299-306 (2015).

23. Morello L. More cuts loom for US science. Nature 501, 147-148 (2013). 
